\title{
Adolf Kussmaul (1822-1902), su biografía y descripciones
}

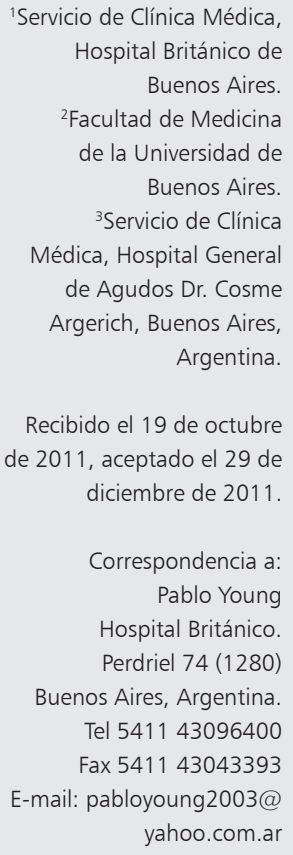

Recibido el 19 de octubre de 2011, aceptado el 29 de diciembre de 2011.

Correspondencia a: Pablo Young Hospital Británico. Perdriel 74 (1280)

Buenos Aires, Argentina. Tel 541143096400 Fax 541143043393

E-mail: pabloyoung2003@ yahoo.com.ar

\author{
PABLO YOUNG ${ }^{1}$, BÁRBARA C. FINN ${ }^{1}$, JULIO E. BRUETMAN ${ }^{1}$, \\ ALFREDO BUZZI $^{2}$, MARCELO ZYLBERMAN ${ }^{3}$
}

\section{The outstanding achievements of Adolf Kussmaul}

Adolf Kussmaul was born in Graben, close to Karlsruhe, Germany, on February 22, 1822. He graduated at Heidelberg University in 1845 and he received his medical degree in 1855. He made original descriptions, such as the ophthalmoscope, the gastroscope or endoscope, and Kussmaul signs, besides the respiration named after him. He was the first to perform a thoracocenteses, peritoneal and gastric lavages. He rediscovered progressive bulbar paralysis (previously described by Guillaume Duchenne in 1861), polyarteritis nodosa (which was described by Karl von Rokitansky en 1852), dyslexia, (described by Thomas Willis in 1672) and pulsus paradoxus named after him, but that was reported by Richard Lower in 1669. During his 80 years of life, he wrote about different issues and topics (cardiology, rheumatology, endocrinology, gastroenterology, psychiatry, and neurology). Kussmaul was a student and professional without limits in his observation capacity. He preached with his own example in pursuit of science: observation, hypothesis, experimentation and analysis.

(Rev Med Chile 2012; 140: 538-544).

Key words: History of Medicine; Medical history taking; Pericardium; Physical examination.

\section{Perspectiva histórica}

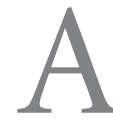

dolf Kussmaul nació en Graben, cerca de Karlsruhe en Baden, Alemania, el 22 de febrero de 1822 (Figura 1) ${ }^{1}$. Heredó la vocación de médico de su padre y su abuelo, que habían sido cirujanos del ejército alemán. Inició sus estudios de medicina en 1840, a la edad de 18 años, en la Universidad de Heidelberg y se graduó de cirujano, clínico y ginecólogo, en 1845.

Al ingresar a la Universidad, tuvo como maestros a médicos de la talla de Jakob Henle (1809-1885) (quien describió el “asa” que aún hoy lleva su nombre), de Franz Karl Nägele (1778-1851), famoso por su regla para calcular la fecha probable de parto, y al profesor Johannes Müller (1801-1858), gran impulsor de la fisiología en Alemania, conocimientos compilados, en su tratado de fisiología humana. Müller describió la sensibilidad de la retina a los estímulos cromáticos, la ley de la energía específica de los sentidos, y los conductos en el embrión, que llevan su nombre. Las investigaciones de Müller en la fisiología ocular incentivaron a Kussmaul en ese tema, por lo que desarrolló una tesis relacionada con los cambios de color en la retina. También inventó, en 1845, mientras era aún un estudiante, el primer oftalmoscopio complejo que, años después, Hermann Ludwig von Helmholtz (1821-1894) reformó y así, su inventor fue el pionero de la oftalmología moderna ${ }^{2}$. En 1847 visitó Viena y conoció a Joseph Skoda (1805-1881) a quien admiró por su excelencia en el examen físico, y en el Hospital General de esa ciudad, conoció a Ferdinand Ritter von Hebra (1816-1880) y sus primeras incursiones en la dermatología, continuando en este campo, en Praga, bajo la dirección de Johannes Oppolzer (1808-1871) $)^{3}$.

Entre 1848 y 1849, siguiendo los pasos de su padre, Kussmaul se enroló en el ejército como cirujano, durante la campaña militar alemana contra Dinamarca, en Schleswig-Holstein. Una 


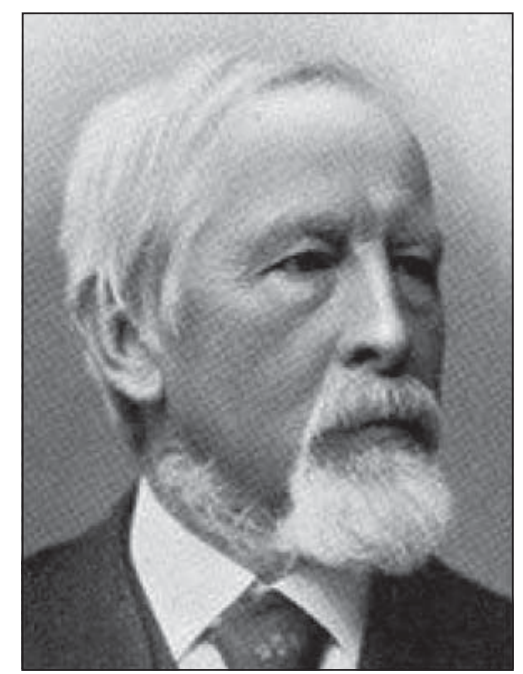

Figura 1. Dr. Adolf Kussmaul. Tomado de: www.anaesthesiamcq.com/images/Kussmaul.jpg.

vez terminado el enrolamiento se casó, en 1850, y ejerció la práctica privada hasta 1853 , año en que trabajó en su tesis doctoral, en la Universidad de Würzburg, junto a Rudolf Virchow (1821-1902). En 1855, recibió el título de Doctor en Medicina. Entre 1859 y 1863 ejerció la docencia en las Universidades de Heidelberg y Erlangen. En la Universidad de Erlangen trabajó al lado del patólogo Friedrich Albert von Zenker (1825-1898) y con el internista Hugo Wilhelm von Ziemssen (1829-1902). En 1866, Zenker y Ziemssen fundaron la revista "Deutsches Archiv für klinische Medizin" (Archivos alemanes de medicina interna). Kussmaul y Rudolf Maier publicaron en el volumen inaugural de esta revista, los primeros casos bien estudiados de periarteritis nodosa. Posteriormente, fue profesor de medicina interna en la Universidad de Freiburg, hasta 1877, y en la de Estrasburgo hasta $1888^{4}$.

\section{Contexto político y científico en el siglo XIX}

La "Alemania" de 1822, cuando nació Kussmaul, era en realidad la "Confederación Germánica" formada a partir del imperio austriaco, los reinos de Baviera, Hanover, Prusia, Sajonia y varios ducados, principados y ciudades libres donde estaba el Gran Ducado de Baden, al cual pertenecía la ciudad natal de Kussmaul. Una reforma de la constitución danesa y la disputa por Schleswig-Holstein causó la guerra conocida como "de los ducados" (1864), y después la guerra de las siete semanas (1866), por las cuales Prusia se aseguró la hegemonía germánica. El Imperio Alemán se fundó el 18 de enero de 1871, tras la victoria de Prusia en la guerra franco-prusiana y se consiguió la unificación de los diferentes estados alemanes en torno a Prusia, excluyendo a Austria.

Los filósofos del idealismo alemán (Kant, Fichte, Hegel), científicos, artistas y literatos de todos los ámbitos (Goethe, los hermanos Humboldt, los hermanos Grimm, Wagner, Beethoven, Schumann, Brahms, Mendelssohn, Meyerbeer, etc.) se implicaron en los diferentes movimientos sociales y políticos que, con el tiempo y tras muchas alternativas, llevaron a la unificación alemana.

El positivismo es un concepto polisémico que encierra diversos sentidos dentro de una connotación histórica, epistemológica y filosófica, y que alude a una corriente filosófica, o a una tendencia científica y cultural, que se desarrolló en la Europa decimonónica, a partir de las ideas de Augusto Comte (1798-1857), Emile Littré (1801-1881) y John Stuart Mill (1806-1873), entre otros. Pusieron de manifiesto la importancia del método científico y de la ciencia como fenómeno cognitivo y social, que posibilitan un ascenso inevitable hacia el progreso material y moral ${ }^{5}$.

En el siglo XIX la medicina se relacionó directamente con los fenómenos migratorios, los hacinamientos en las ciudades y las precarias condiciones de vida de la clase trabajadora, propios de la revolución industrial. Su consecuencia fue la proliferación de enfermedades infecciosas (sífilis, tuberculosis) o relacionadas con la mala alimentación (pelagra, raquitismo, escorbuto). Dichas problemáticas son cruciales para entender el origen de la medicina social de Rudolf Virchow y el sistema de salud pública de Edwin Chadwick, que darían lugar a la actual medicina preventiva. La misma Revolución Industrial, con el agregado de las numerosas guerras y revoluciones, generarían un desarrollo científico generalizado que contribuiría a la instauración de condiciones técnicas para el triunfo de la asepsia, la anestesia y la cirugía. En el año 1848 comenzó a emplearse el éter para sedar a los pacientes antes de la cirugía, y a finales de este siglo Luis Pasteur, Robert Koch y Joseph Lister demostraron inequívocamente la naturaleza etiológica de los procesos infecciosos, mediante la teoría microbiana. 
Este siglo vió nacer la teoría de la evolución de las especies, de Charles Darwin, expresión antropológica del positivismo científico que le es propio. La realidad puede medirse, comprenderse y predecirse mediante leyes, que a su vez van siendo corroboradas por los sucesivos experimentos. Por ese camino avanzaron la astronomía (Laplace, Foucault), la física (Poincaré, Lorentz), la química (Dalton, Gay-Lussac, Mendeleiev) y la genética de Gregor Mendel.

La figura médica por excelencia de este siglo fue Rudolf Virchow, quien postuló la teoría de "toda célula proviene de otra célula" y explicó a los organismos vivos como estructuras formadas por células. En Viena, Joseph Skoda y Carl von Rokitansky fundaron la Escuela Moderna de Medicina. Como se mencionó previamente, con estos tres médicos también estudió Kussmaul.

En el siglo XIX compitieron y se complementaron dos concepciones médicas capitales: el criterio anátomo-clínico, encumbrado por la obra de René Théophile Hyacinthe Laënnec (1781-1826), y el criterio fisiopatológico, que recibió el vigoroso impulso de Claude Bernard (1813-1878) y, con él, la prédica del razonamiento experimental, base de la investigación biológica. También a finales de siglo un físico alemán, Wilhelm Röntgen, descubrió los Rayos X.

La situación política a mediados del siglo XIX fue el principal determinante de la emigración de familias alemanas que colonizaron el sur de Chile ${ }^{5}$. Entre ellas llegaron profesionales ilustres, como el primer Editor y fundador de la Revista Médica de Chile, el Dr. Germán Schneider (1820-1884), quien se formó como médico en el ambiente científico en que trabajó Kussmaul ${ }^{6}$. Adolf von Strümpell (1853-1925), uno de los fundadores de la neurología moderna, a través de su discípulo Christofredo Jakob (1866-1956) influyó de manera determinante sobre la tradición neurobiológica argentino-germana. Otro ejemplo indirecto es el del médico argentino Bernardo Houssay (18871971), que en 1907, luego de leer "Introduction a l'etude de la médécine experimentale" de Claude Bernard, decidió ser fisiólogo y en 1947 obtuvo el Premio Nobel de Medicina por su trabajo sobre la influencia del lóbulo anterior de la hipófisis en la distribución de la glucosa en el cuerpo, de importancia para el desarrollo de la diabetes, siendo el primer argentino y latinoamericano laureado en Ciencias.

\section{Descripción de la poliarteritis nodosa}

Se ha analizado detalladamente la historia de la poliarteritis nodosa (PAN), a raíz de la información publicada por Hans Eppinger (1846-1916), profesor de patología y anatomía en Graz (Austria), quien escribió un artículo sobre anatomía y patología de los aneurismas en 1887 y mencionó los estudios de los precursores como Morgagni, Monro, Hunter, Palleta, Broco, Lebert, Virchow, Scarpa, Lobstein, y Cruveilhier ${ }^{7}$. El estudio de Dickson de la Universidad de Edinburgo, una de las más importantes del mundo en el siglo XVII, introdujo el término de PAN en la literatura inglesa, en $1908^{8}$. Distinguió la periarteritis nodosa, que sólo compromete la capa externa de las arterias como la llamaba el patólogo Maier, ${ }^{90}$ y la denominó poliarteritis nodosa aguda, como lo sugirió Enrico Ferrari, de Trieste, en 1903, debido a que esta arteritis se asociaba a nodulosis que comprometía las arterias de mediano calibre, debido a un proceso inflamatorio localizado, destructivo y proliferativo que ocurre en toda la pared de los vasos $^{11}$. La doctora Pearl M. Zeek, de la Universidad de Cincinnati, analizó en períodos históricos la descripción de esta vasculitis adjudicando su primera descripción a Karl von Rokitansky (1804-1878) quien la reportó en 1852 (Figura 2 y 3$)^{12-14}$. La Dra. Zeek realizó la primera clasificación de las vasculitis en 1952 y diferenció la PAN de las vasculitis por hipersensibilidad, conocidas posteriormente como vasculitis leucocitoclástica cutánea y en la actualidad como vasculitis cutánea de pequeños vasos ${ }^{15}$.

El artículo que generó la apertura del conocimiento de la PAN, fue la descripción clásica de Adolf Kussmaul y del patólogo Rudolf Robert Maier (1824-1888), en Freiburg9. El paciente Carl Seufarth, de 27 años, sastre de profesión, consultó por presentar síntomas agudos de fiebre, mialgias, mononeuritis múltiple, dolor abdominal y proteinuria. Fue hospitalizado en el servicio del profesor Kussmaul, y al salir del hospital, fue aprehendido por la policía por pedir limosna. Fue hospitalizado nuevamente durante un mes, y falleció por su cuadro clínico general; tres días antes de su muerte presentó nódulos en el tejido subcutáneo a nivel de la piel del tórax y abdomen. La autopsia mostró nódulos a lo largo de las arterias del hígado, corazón, coronarias, mesenterio, estómago, riñón, bazo, diafragma, 
músculos y tejido celular subcutáneo. El examen microscópico mostró que la íntima de las arterias comprometidas estaba preservada, mientras que la capa media y la adventicia tenían inflamación importante. A nivel renal, observaron cambios parecidos a la enfermedad de Bright aguda ${ }^{16}$, con compromiso de las arterias interlobulares. Kussmaul y Maier atribuyeron los cambios en las arterias a un proceso inflamatorio que afectaba las cubiertas perivasculares, especialmente la media y la adventicia y observaron que el proceso inflamatorio que comprometía las arterias podía involucrar los tejidos musculares y conectivos. Los autores especularon que estos cambios pudiesen estar asociados al nematodo estrongiloide; por ello, el primer reporte de su caso, se publicó bajo el título de "Aneurysma verminosum hominis",

Los autores describieron un segundo paciente, Landolin Faist, de 28 años, quien consultó por mialgias y debilidad muscular de 3 días de evolu-

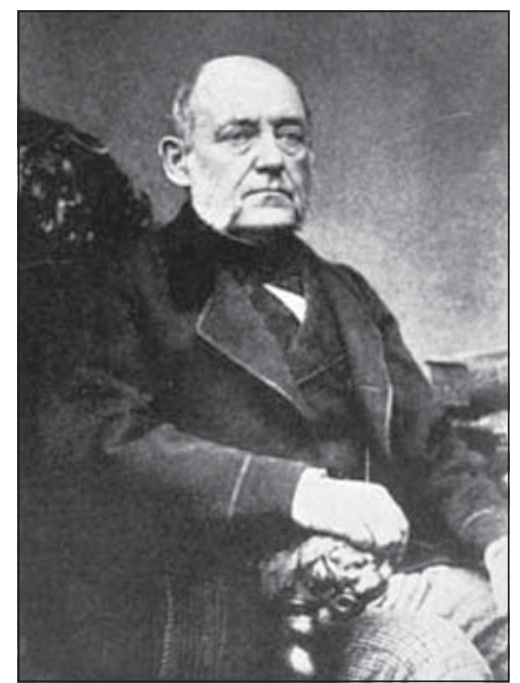

Figura 2. Dr. Carl von Rokitansky Tomado de: www.historiadelamedicina.org/imagenes/rokitansky.jpg

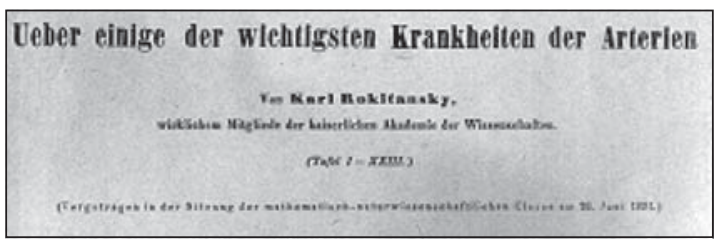

Figura 3. Facsímil del libro donde se hace la descripción inicial de la PAN por Rokitansky. ción y compartió el cuarto con Seufarth ${ }^{10}$. Presentó edema de miembros inferiores, en el uroanálisis se le documentó proteinuria, pero no hematuria. Este paciente fue el primero en la historia de la medicina en quien, estando vivo, se le practicó una biopsia, para definir un diagnóstico de vasculitis. En la biopsia se observó degeneración granular del tejido muscular pero no vasculitis. Kussmaul y Maier, al parecer, al publicar sus dos artículos no conocían las publicaciones de Rokitansky, en donde ya describía esta entidad, o si lo conocían no lo mencionaron ${ }^{17,18}$. Es muy probable que Kussmaul conociera el trabajo de Rokitansky, pero no lo mencionó porque Kussmaul no lo consideró un buen maestro. Kussmaul estudió en Viena con el profesor Rokitansky hasta 1847 y el paciente reportado por Rokitansky, Wenzel Plohner, lo consultó a éste en 1848. Históricamente los dos artículos de Kussmaul y Maier son las descripciones clásicas del cuadro clínico y de la patología, fueron los primeros en mencionar el nombre, por lo tanto, no hay duda sobre el mérito de estos dos médicos y sobre los aportes para el conocimiento de esta enfermedad ${ }^{19}$. A la luz de lo expresado previamente deberíamos llamar a esta entidad, enfermedad de Rokitansky-Kussmaul-Maier.

Karl von Rokitansky, el gran patólogo vienés, en su libro "Ueber einige der wichtigsten Krankheiten der Arterien" (sobre algunas de las más importantes enfermedades de las arterias), en 1852 informó sobre la presencia de lesiones aneurismáticas con nódulos en las arterias de una autopsia de un paciente de 23 años que trabajaba como zapatero. La historia de Wenzel Plohner, se remonta al 6 de noviembre de 1848, cuando fue admitido al hospital por diarrea, enterorragia, fiebre y dolor abdominal de 5 días de evolución. El paciente murió casi dos meses después de los síntomas, el 12 de enero de 1849; Rokitansky le practicó la autopsia, pero no examinó la histología; a grosso modo, analizó el corazón, las arterias coronarias, el hígado y el árbol mesentérico arterial; le llamó la atención la presencia de aneurismas en el sistema vascular arterial, con la excepción de la aorta, sus ramas principales y las arterias cerebrales. Describió los aneurismas cerca de las ramificaciones y la disección de los aneurismas por rotura a nivel de la pared de la íntima y de la media. Treinta y cinco años después, Hans Eppinger remarcó los aspectos macro y microscópicos de los especímenes del intestino delgado y de las 
arterias coronarias. Eppinger analizó la patogénesis de los aneurismas y contradijo a Rokitansky, al observar que toda la pared de los aneurismas estaba comprometida y no sólo la íntima. Al revisar la histología, describió un engrosamiento de la íntima con depósitos celulares y disrupción de todas las capas (medias, elásticas y adventicias) con múltiples focos de células y fibras. Hans Eppinger clasificó los aneurismas en congénitos, parasíticos o infecciosos y simples ${ }^{7}$.

\section{Otras descripciones}

La producción científica de Kussmaul fue excepcional y diversa (Tabla 1). Describió la parálisis bulbar progresiva, enfermedad previamente descripta por el neurológo frances Guillaume Duchenne (1806-1875), en 1861, denominada por Jean Martin Charcot (1825-1863) parálisis labioglosolaringea (involucra específicamente los pares craneales IX, X, y XII) y que fuera asociada a la esclerosis lateral amiotrófica (conocida como enfermedad de Charcot) por Joseph Jules Dejerine (1849-1917) en $1883^{20}$.

Estudió el mecanismo de los tragadores de espadas (faquires), tradición que llevaba más de 4.000 años (Figura 4), para el desarrollo del endoscopio rígido (gastroscopía) en 1868, por lo cual se lo considera el precursor de la endoscopía; ese primer intentó falló, por falta de una apropiada fuente de luz ${ }^{21}$.

Introdujo, también, el uso de lavado gástrico (para eliminar venenos del estómago y como procedimiento previo a cirugías gastrointestinales), y fue el primero en publicar un caso de embolia mesentérica. También fue el primero en utilizar la toracocentesis y hacer un lavado peritoneal. Describió lo que se conoce como "ondas peristálticas de lucha, de Kussmaul”, en el síndrome pílórico.

Fue un suspicaz observador de los trastornos del lenguaje. Su libro sobre afasia era un clásico en el tema y quizás su aporte más significativo; realizó la primera descripción de un caso de dislexia en 1877 (aunque ya había sido descripta por Thomas Willis en 1672), a la que denominó "ceguera verbal”, y también, detalló el mutismo selectivo, llamado por mucho tiempo "afasia de Kussmaul", que se trata de un desorden de ansiedad severo de la niñez donde la persona es capaz de hablar en ciertas situaciones y con ciertas personas ${ }^{22}$.

Tabla 1. Cronología de las descripciones de Adolf Kussmaul

\begin{tabular}{|c|c|}
\hline Año & $\begin{array}{l}\text { Descripción de enfermedad, entidad, } \\
\text { signo o procedimiento }\end{array}$ \\
\hline 859 & $\begin{array}{l}\text { la salivación durante la administra } \\
\text { rio }\end{array}$ \\
\hline 861 & $\begin{array}{l}\text { parálisis bulbar progresiva, enfermedad } \\
\text { previamente por Duchenne }\end{array}$ \\
\hline 866 & ifermedad de \\
\hline 868 & Describió el endoscopio rígido (gastroscopía) \\
\hline 869 & Utilizó el lavado gástrico \\
\hline 1869 & Describió la embolia mesentérica \\
\hline 869 & $\begin{array}{l}\text { Describió y utilizó la toracocentesis y el lavado } \\
\text { peritoneal }\end{array}$ \\
\hline 870 & $\begin{array}{l}\text { Describió las ondas peristálticas de lucha de } \\
\text { Kussmaul }\end{array}$ \\
\hline 1873 & $\begin{array}{l}\text { paradójico, descripto previa- } \\
\text { _ower }\end{array}$ \\
\hline 1874 & Describió la respiración de Kussmaul \\
\hline 1875 & $\begin{array}{l}\text { Describió el signo de Kussmaul o ingurgitación } \\
\text { yugular durante la inspiración }\end{array}$ \\
\hline 1877 & $\begin{array}{l}\text { Describió la dislexia, previamente descripta por } \\
\text { Thomas Willis }\end{array}$ \\
\hline 1877 & $\begin{array}{l}\text { ó el mutismo selectivo, llamado "Afasia } \\
\text { maul" }\end{array}$ \\
\hline 1879 & $\begin{array}{l}\text { Describió el signo de Kussmaul o emanación de } \\
\text { pus por el orificio del conducto de Wharton debida } \\
\text { a la infección secundaria que acompaña a la litiasis } \\
\text { submaxilar luego de la compresión de la glándula }\end{array}$ \\
\hline
\end{tabular}

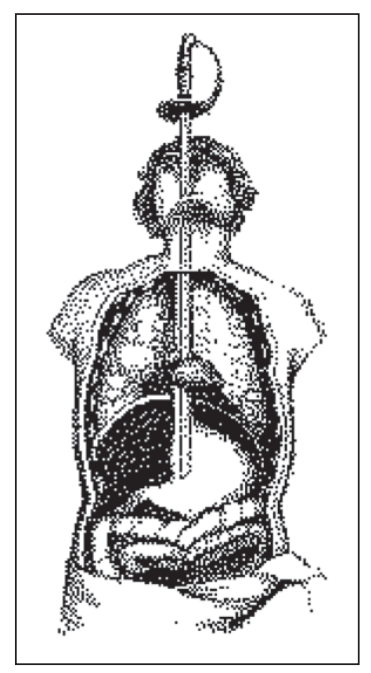

Figura 4. Imagen de Faquir. Tomado de: http://www. swordswallow.org/news.php 
Entre sus informes clínicos, que son clásicos en la literatura médica, están las descripciones del pulso paradójico que lleva su nombre, publicado el 22 de septiembre de 1873 y que describió como una marcada disminución de la amplitud de la onda del pulso arterial durante la inspiración y se define actualmente como el descenso de la presión arterial sistólica mayor a $10 \mathrm{mmHg}$ durante la inspiración. Este signo había sido descripto previamente por Richard Lower (1631-1691), en el año 1669, y se asocia a pericarditis constrictiva, hipertensión pulmonar aguda, asma, enfermedad pulmonar obstructiva crónica, entre otras ${ }^{1,16,23-25}$. Por ello proponemos el nombre de pulso de Lower-Kussmaul.

Irónicamente, Kussmaul desarrolló una pericarditis en 1846, secundaria a un reumatismo articular agudo o fiebre reumática o enfermedad de Bouillaud, descripta por Jean-Baptiste Bouillaud (1796-1881), en $1836^{13,319,25}$.

La respiración de Kussmaul, peculiar respiración descripta como "hambre de aire", es la hiperventilación secundaria a la acidosis metabólica severa que ocurre en la cetoacidosis diabética o insuficiencia renal, y su descripción data de $1874^{26}$.

Se llama signo de Kussmaul al incremento de la presión venosa yugular e ingurgitación que se observa durante la inspiración, aproximadamente en $33 \%$ de las pericarditis constrictivas, en 33-100\% de los infartos de ventrículo derecho, en la cardiomiopatía restrictiva, en la estenosis tricuspídea e hipertensión pulmonar. Es de remarcar que este signo está ausente en el taponamiento cardíaco ${ }^{27}$.

Por último, se conoce como signo de Kussmaul a la emanación de pus por el orificio del conducto de Wharton debida a la infección secundaria que acompaña a la litiasis submaxilar luego de la compresión de la glándula.

En 1845 estudió los cambios de color en el ojo, sobre la influencia de la circulación en los movimientos del iris y en la relación entre la anemia y las convulsiones epilépticas. En 1859 escribió una monografía sobre la psicología del lactante. También describió la salivación durante la administración de mercurio. Fue el primero en emplear el bismuto en el tratamiento de la úlcera gástrica. Publicó su autobiografía con el título de "Recuerdos juveniles de un médico anciano".

Kussmaul fue un hombre meticuloso y preciso, famoso por quejarse que ninguno de sus compañeros sabía escribir bien el alemán. Escribió

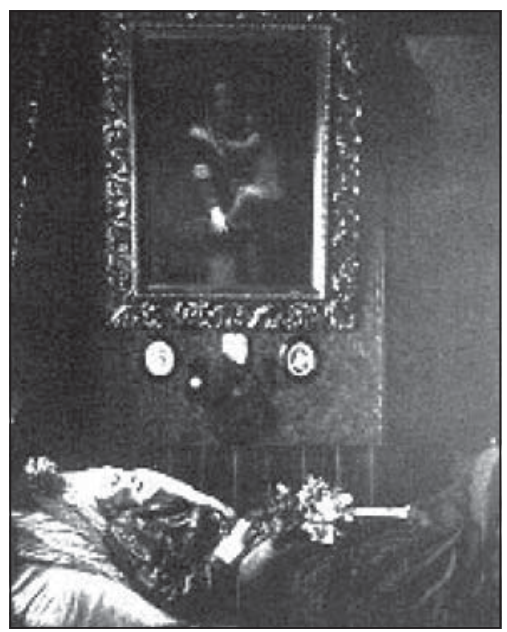

Figura 5. Lecho de muerte del Dr. Adolf Kussmaul. Tomado de: http://en.wikipedia.org/wiki/Adolph_Kussmaul

poemas satíricos para una revista semanal con el seudónimo de Gottlieb Biedermeier, un poeta imaginario y poco sofisticado que, finalmente, llegó a simbolizar los valores y los gustos de la burguesía alemana de principios del siglo XIX; fiable, trabajador, pero aburridamente poco imaginativo (como el estilo de muebles Biedermeier; Bieder es una palabra alemana que significa cotidiano, simple y Meier, o Meyer, es un apellido alemán frecuente) ${ }^{28}$.

Adolf Kussmaul, falleció por un infarto a la edad de 80 años, el 28 de mayo de 1902, en Heidelberg, Alemania (Figura 5).

En sus 80 años de vida, Adolf Kussmaul realizó descubrimientos más allá de las capacidades de un solo hombre. Ejemplifica un modelo de estudiante y profesional sin límites en su capacidad de observación. Predicó con el ejemplo en pos de la ciencia: observación, hipótesis, experimentación y análisis, aportando a la medicina con sus descubrimientos.

\section{Referencias}

1. Bilchick KC, Wise RA. Paradoxical physical findings described by Kussmaul: pulsus paradoxus and Kussmaul's sign. Lancet 2002; 359: 1940-2.

2. Mark HH. The first ophthalmoscope? Adolf Kussmaul 1845. Arch Ophthalmol 1970; 84: 520-1.

3. Matteson EL, Kluge FJ. Think clearly, be sincere, act calmly: Adolf Kussmaul (February 22, 1822-May 28, 
1902) and his relevance to medicine in the 21st century. Curr Opin Rheumatol 2003; 15: 29-34.

4. Breathnach CS, Westphal W. Adolf Kussmaul (18221902): from youth to maturity under the influence of Virchow. J Med Biogr 2003; 11: 55-61.

5. Cruz-Coke R. [The evolution theory in the medical sciences in Chile]. Rev Med Chile 1994; 122: 211-4.

6. Reyes H, Kauffmann R, Andresen M. [A tribute to Germán Schneider, MD, first editor of Revista Médica de Chile]. Rev Med Chile 1997; 125: 761-4.

7. Eppinger H. Pathogenesis (Histogenesis und Aetiologie) der Aneurysmen einschliesslich des Aneurysma equiverminusum. Arch Klin chir 1887; 35: 1-563.

8. Dickson WEC. Polyarteritis acuta nodosa and periarteritis nodosa. J Pathol Bact 1908; 12: 31-57.

9. Kussmaul A, Maier R. Aneurysma verminosum hominis vorläufige Nachricht. Deutsch Arch Klin Med 1866; 1 : 125-6.

10. Kussmaul A, Maier R. Ueber einen bisher nicht beschriebene eigenthümliche Arterienekrankung (periarteritis nodosa), die mit Morbus Brightii und rapid fortschreiteder allgemeiner Muskellähmung einhergeht. Deutch Arch Klin Med 1866; 1: 484-518.

11. Ferrari E. Ueber Polyarteritis acute (sogennante periarteritis nodosa) und ihre Beziehungen zur Polymyositis und Polyneuritis acuta. Beitr pathol Anat allg pathol 1903; 34: 350-86.

12. Zeek PM. Periarteritis nodosa: critical review. Am J Clin Pathol 1952; 22: 777-90.

13. Zeek PM. Periateritis nodosa and other forms of necrotizing angiitis. N Engl J Med 1953; 248: 764-72.

14. Rokitansky K. Ueber einige der wichtigsten Krankheinten der Arterien. Vienna: Kaiserlich-Koeniglichen Hof-und Staatsdruckerei, 1852; 4: 1-72.

15. Koutkia P, Mylonakis E, Rounds S, Erickson A. Leucocytoclastic vasculitis: an update for the clinician. Scand
J Rheumatol 2001; 30: 315-22. (Review).

16. Zylberman M, Young P. [Pericardial disease: from antiquity to the ultrasound]. Gac Med Mex 2010; 146: 348-53.

17. Matteson EL. History of early investigation in polyarteritis nodosa. Arthritis Care Research 1999; 12: 294-302.

18. Tesar V, Kazderová M, Hlavácková L. Rokitansky and his first description of polyarteritis nodosa. J Nephrol 2004; 17: 172-4.

19. Gamarra AI, Restrepo Suárez JF, Valle R, Osorio E, Bolaños A, Méndez O, et al. Historia de las vasculitis. Rev Colombiana Reumatol 2002; 9: 87-121.

20. Lapiedra RC, Moreno López LA, Esparza Gómez GC. Progressive bulbar palsy: a case report diagnosed by lingual symptoms. J Oral Pathol Med 2002; 31: 277-9.

21. Pellicano R, Bocus P, De Angelis C. Adolf Küssmaul, the sword eater and modern challenges of digestive endoscopy. Minerva Gastroenterol Dietol 2011; 57: 109-10.

22. Henderson VW. Chapter 37: alexia and agraphia. Handb Clin Neurol 2010; 95: 583-601.

23. Kussmaul A. Über schwielige Mediastino-Perikarditis und den paradoxen Puls. Berliner klinische Wochenschrift 1873, 10: 433-5.

24. Abu-Hilal MA, Mookadam F. Pulsus paradoxus; historical and clinical perspectives. Int J Cardiol 2010; 138: 229-32.

25. Johnson SK, Naidu RK, Ostopowicz RC, Kumar DR, Bhupathi S, Mazza JJ, et al. Adolf Kussmaul: Distinguished clinician and medical pioneer. Clinical Medicine and Research 2009; 7: 107-12.

26. Kussmaul A. Zur lehre vom diabetes mellitus. Dtsch Arch Klin Med 1874; 14: 1-46.

27. Minagar A, Weiner WJ. Adolf Kussmaul and his respiratory sign. J Med Biogr 2001; 9: 181-3.

28. Fodor P. [Satirical poems on physicians (Adolf Kussmaul)]. Orv Hetil 1971; 112: 458-61. 